ROCKY MOUNTAIN

JOURNAL OF MATHEMATICS

Volume 24, Number 1, Winter 1994

\title{
ASYMPTOTICS AND OSCILLATION FOR FIRST ORDER NEUTRAL FUNCTIONAL DIFFERENTIAL EQUATIONS
}

\author{
R.M. MATHSEN, XU YUANTONG AND WANG QIRU
}

Dedicated to Paul Waltman on the occasion of his 60th birthday

\begin{abstract}
The asymptotic behavior of all nonoscillatory solutions of some linear first order neutral delay differential equations is studied. A solution to one conjecture by Grammatikopoulos, Grove and Ladas is given and part of a second conjecture is proved. A test for oscillation in terms of the coefficients, the delay and the advance is given for neutral equations of mixed type.
\end{abstract}

1. Introduction. In this paper we deal with the first order linear neutral functional differential equation

$$
\frac{d}{d t}[y(t)+p y(t-\tau)]+q y(t-\sigma)=0
$$

for $t \geq t_{0}$ where $q \neq 0, p, \tau$ and $\sigma$ are real numbers.

From the point of view of applications, NFDEs appear as models of electrical networks which contain lossless transmission lines. Such networks arise, for example, in high speed computers where lossless transmission lines are used to interconnect switching circuits (see [2, 11]). Concerning existence, uniqueness and continuous dependence of solutions, see Hale [7] and Driver [3, 4].

As usual, a solution of equation (1) is called oscillatory if it has arbitrarily large zeros and nonoscillatory if it is eventually positive or negative.

Recently, Grammatikopoulos, Grove and Ladas in [5] studied asymptotic behavior of nonoscillatory solutions of NFDEs (1) under various

Received by the editors on September 2, 1993

AMS 1991 Mathematics Subject Classification. 34K25, 34K40.

Key Words. Neutral equation, asymptotic behavior, oscillation.

Part of this paper was written while the first and second authors were visiting the Applied Mathematics Institute at the University of Alberta.

Research of the second author supported in part by the Canada-China Scholarly Exchange Program.

Copyright (c)1994 Rocky Mountain Mathematics Consortium 
conditions on $q, \tau$ and $p$. Their results are summarized in the table below. Here $y(t)$ is a nonoscillatory solution of (1) with $\lim _{t \rightarrow \infty} y(t)$ the entries in the table for the various cases.

TABLE 1.

\begin{tabular}{|c|c|c|c|c|c|c|c|}
\hline$q$ & $\tau$ & $p<-1$ & $p=-1$ & $-1<p<0$ & $0 \leq p<1$ & $p=1$ & $1<p$ \\
\hline+ & + & $\pm \infty$ & no nonoscil- & 0 & 0 & 0 & 0 \\
+ & 0 & $\pm \infty$ & latory solutions & 0 & 0 & 0 & 0 \\
\hline+ & - & $\pm \infty$ or 0 & $?$ & $?$ & 0 & 0 & 0 \\
\hline- & + & $?$ & $?$ & $\pm \infty$ or 0 & $\pm \infty$ & $?$ & $?$ \\
\hline- & 0 & 0 & no nonoscil- & $\pm \infty$ & $\pm \infty$ & $\pm \infty$ & $\pm \infty$ \\
- & - & 0 & latory solutions & $\pm \infty$ & $?$ & $?$ & $\pm \infty$ \\
\hline
\end{tabular}

For the missing cases (marked by "?"), two conjectures were given in $[5]$.

Conjecture 1. Suppose $p<0$ and $q \tau<0$. Let $y(t)$ be $a$ nonoscillatory solution of (1). Then $\lim _{t \rightarrow \infty} y(t)$ must be $-\infty, 0$ or $+\infty$.

Conjecture 2. Suppose $p>0, q<0$ and $\tau \neq 0$. Let $y(t)$ be $a$ nonoscillatory solution of (1). Then $\lim _{t \rightarrow \infty} y(t)$ equals either $-\infty$ or $+\infty$.

In this paper we shall discuss these two conjectures. In Section 2 we state some lemmas which are useful in the sequel. In Section 3 we prove Conjecture 1. In Section 4 we prove Conjecture 2 in the case that $p=1$. It has recently been shown in [8] that Conjecture 2 is false in the case that $p>1, q<0$, and $\tau>0$. However, additional assumptions on $p, q$ and $\tau$ do give the asymptotic behavior claimed in Conjecture 2. These results and a short discussion of oscillation when $\sigma<0$ appear in Section 4. A concluding discussion and some examples appear in Section 5. 
2. Some basic lemmas. In this section we restate some lemmas from [5] which are useful in our proofs of the conjectures.

Lemma 1. Suppose $y(t)$ is an eventually positive solution of Equation (1). Set

$$
z(t)=y(t)+p y(t-\tau) .
$$

Then

$$
\lim _{t \rightarrow \infty} z(t)= \begin{cases}0 \text { or } \infty, & \text { if } q<0 \\ -\infty \text { or } 0, & \text { if } q>0\end{cases}
$$

Lemma 2. Suppose either that $q \tau \geq 0$, or else that $q \tau<0$ and $p \geq 0$. Let $y(t)$ be an eventually positive solution of (1). Set

$$
z(t)=y(t)+p y(t-\tau) .
$$

Then

$$
\lim _{t \rightarrow \infty} z(t)= \begin{cases}-\infty, & \text { if } p<-1, q>0, \tau \geq 0 ; \\
0, & \text { if }\left\{\begin{array}{l}
p<-1, q<0, \tau \leq 0, \\
-1<p, q>0, \tau \geq 0, \\
0 \leq p, q>0, \tau<0
\end{array}\right. \\
\infty, \quad \text { if }\left\{\begin{array}{l}
-1<p, q<0, \tau \leq 0, \\
0 \leq p, q<0, \tau>0
\end{array}\right.\end{cases}
$$

Lemma 3. Suppose $p \neq 0$ as well as $q \neq 0$. Then $y(t)$ satisfies the NFDE (1) if and only if $y(t)$ satisfies the NFDE

$$
\frac{d}{d t}\left[y(t)+\frac{1}{p} y(t-(-\tau))\right]+\frac{q}{p} y(t-(\sigma-\tau))=0 .
$$

\section{Proof of Conjecture 1.}

Theorem 1. Suppose $p<0$ and $q \tau<0$. Let $y(t)$ be a nonoscillatory solution of (1). Then

$$
\lim _{t \rightarrow \infty} y(t)=-\infty \quad \text { or } \lim _{t \rightarrow \infty} y(t)=0 \quad \text { or } \lim _{t \rightarrow \infty} y(t)=+\infty .
$$


Proof. Since the negative of a solution of (1) is also a solution of (1), it suffices to consider an eventually positive solution $y(t)$ of (1). Suppose then that $y(t)>0$ for $t \geq t_{0}$. We first consider the case that $q<0$ and $\tau>0$. Let $z(t):=y(t)+p y(t-\tau)$. Then $z^{\prime}(t)=-q y(t-\sigma)$ and, by Lemma $1, z(t)$ increases monotonically to 0 or $\infty$ as $t \rightarrow \infty$. If $\lim _{t \rightarrow \infty} z(t)=\infty$, then $y(t)>y(t)+p y(t-\tau)=z(t)$, so $\lim _{t \rightarrow \infty} y(t)=\infty$ also. We now suppose that $\lim _{t \rightarrow \infty} z(t)=0$ and show that $\lim _{t \rightarrow \infty} y(t)=0$ as well. We consider the three subcases $p>-1, p=-1$ and $p<-1$.

a) Assume $-1<p<0$. Note that $y(t)+p y(t-\tau)=z(t)<0$, so that $y(t)<-p y(t-\tau)$. Then by iteration we get $y(t+n \tau)<(-p)^{n} y(t-\tau)$ for every positive integer $n$. This implies that $\lim _{n \rightarrow \infty} y(t+n \tau)=0$ uniformly for $\tau \leq t \leq 2 \tau$. Hence, it follows that $\lim _{t \rightarrow \infty} y(t)=0$.

b) Assume $p=-1$. Methods used are similar to ideas in [12]. Integrate $z^{\prime}(t)=-q y(t-\sigma)$ from $t_{0}$ to $t$ and then let $t \rightarrow \infty$. This gives

$$
-z\left(t_{0}\right)=-q \int_{t_{0}}^{\infty} y(s-\sigma) d s .
$$

Thus $y \in L^{1}\left[t_{0}, \infty\right)$ and therefore $z \in L\left[t_{0}, \infty\right)$ also. Since $z(t)$ is monotonically increasing and tends to 0 as $t \rightarrow \infty, \sum_{i=1}^{\infty}|z(t+i \tau)|$ converges uniformly for all $t \in\left[t_{0}, t_{0}+2 \tau\right]$. Hence, for arbitrary $\varepsilon>0$ there is a positive integer $N$ such that

$$
\sum_{i=N+1}^{N+s}|z(t+i \tau)|<\frac{\varepsilon}{2}
$$

for all $t \in\left[t_{0}, t_{0}+2 \tau\right]$ and all positive integers $s$. This implies that, for all $t \in\left[t_{0}, t_{0}+2 \tau\right]$ and all positive integers $s$, we have $|y(t+N \tau)-y(t+(N+s) \tau)|<\varepsilon / 3$. However, $y(t)$ is uniformly continuous on $\left[t_{0}+N \tau, t_{0}+(N+2) \tau\right]$, so there is a $\delta \in(0, \tau)$ such that $\left|y\left(t_{1}\right)-y\left(t_{2}\right)\right|<\varepsilon / 3$ for $t_{1}, t_{2} \in\left[t_{0}+N \tau, t_{0}+(N+2) \tau\right]$ with $\left|t_{1}-t_{2}\right|<\delta$. Now, for arbitrary $t_{1}, t_{2} \geq t_{0}+N \tau$ with $\left|t_{1}-t_{2}\right|<\delta$, we can choose a positive integer $s$ such that $t_{1}=t_{0}+(N+s) \tau+\bar{t}_{1}$, $t_{2}=t_{0}+(N+s) \tau+\bar{t}_{2}$ where $\bar{t}_{1}, \bar{t}_{2} \in[0,2 \tau]$ and $\left|\bar{t}_{1}-\bar{t}_{2}\right|<\delta$. Then we have

$$
\begin{aligned}
\left|y\left(t_{1}\right)-y\left(t_{2}\right)\right| \leq & \left|y\left(t_{0}+(N+s) \tau+\bar{t}_{1}\right)-y\left(t_{0}+\bar{t}_{1}+N \tau\right)\right| \\
& +\left|y\left(t_{0}+\bar{t}_{1}+N \tau\right)-y\left(t_{0}+\bar{t}_{2}+N \tau\right)\right| \\
& +\left|y\left(t_{0}+\bar{t}_{2}+N \tau\right)-y\left(t_{0}+\bar{t}_{2}+(N+s) \tau\right)\right|<\varepsilon
\end{aligned}
$$


Consequently, $y(t)$ is uniformly continuous on $\left[t_{0}+N \tau, \infty\right)$ and hence is uniformly continuous on $\left[t^{0}, \infty\right)$. Thus $z^{\prime}(t)=-q y(t-\sigma)$ is uniformly continuous on $\left[t_{0}, \infty\right)$, and so for arbitrary $\varepsilon>0$ there is a $\delta>0$ such that $\left|z^{\prime}\left(t_{1}\right)-z^{\prime}\left(t_{2}\right)\right|<\varepsilon / 2$ for any $t_{1}, t_{2}, \varepsilon\left[t_{0}, \infty\right)$ with $\left|t_{1}-t_{2}\right|<\delta$. Also, since $\lim _{t \rightarrow \infty} z(t)=0$, there is a $T>0$ such that $|z(t)|<\varepsilon \delta / 8$ for all $t \geq T$. Now for arbitrary $t \geq T$, let $t_{1}=t+\delta / 2$. Then

$$
\left|z^{\prime}(t)-\frac{z(t)-z\left(t_{1}\right)}{t-t_{1}}\right|=\left|z^{\prime}(t)-z^{\prime}(t+\theta \delta / 2)\right|<\frac{\varepsilon}{2}
$$

for some $\theta \in(0,1)$. Therefore,

$$
\left|z^{\prime}(t)\right|<\left|\frac{z(t)-z\left(t_{1}\right)}{t_{1}-t}\right|+\frac{\varepsilon}{2}<\left(\frac{\varepsilon \delta}{8}+\frac{\varepsilon \delta}{8}\right) /\left(\frac{\delta}{2}\right)+\frac{\varepsilon}{2}=\varepsilon .
$$

Hence, $\lim _{t \rightarrow \infty} z^{\prime}(t)=0$ and $\lim _{t \rightarrow \infty} y(t)=0$.

c) Assume $p<-1$. First we show that, for sufficiently large $t \in\left[t_{0}, \infty\right)$, we have

$$
y(t+\tau) \leq y(t)
$$

If not, there is a $t_{1}>t_{0}$ such that $y\left(t_{1}+\tau\right)>y\left(t_{1}\right)$. Since $z(t)$ increases strictly to 0 , it follows that $y\left(t_{1}+2 \tau\right)+p y\left(t_{1}+\tau\right)>y\left(t_{1}+\tau\right)+p y\left(t_{1}\right)$ and thus $y\left(t_{1}+2 \tau\right)-y\left(t_{1}+\tau\right)>-p\left[y\left(t_{1}+\tau\right)-y\left(t_{1}\right)\right]>0$. Hence $y\left(t_{1}+2 \tau\right)>y\left(t_{1}+\tau\right)$ and by iteration $y\left(t_{1}+k \tau\right)>y\left(t_{1}+(k-1) \tau\right)$ for all positive integers $k$. Now $y(t+\tau)-y(t)$ is continuous in $t$ at $t_{1}$, so there must be an $\eta>0$ such that

$$
y(t+\tau)-y(t) \geq\left[y\left(t_{1}+\tau\right)-y\left(t_{1}\right)\right] / 2>0 \quad \text { for all } t \in\left[t_{1}, t_{1}+\eta\right] .
$$

Also, $g:=\min \left\{y(t): t_{1} \leq t \leq t_{1}+\eta\right\}>0$. Then for all positive integers $k$ and all $t \in\left[t_{1}, t_{1}+\eta\right]$ we have $y(t+k \tau)>y(t+(k-1) \tau)>\cdots>$ $y(t) \geq g$. Hence

$$
\int_{t_{1}}^{\infty} y(t) d t=\sum_{k=0}^{\infty} \int_{t_{1}+k \tau}^{t_{1}+(k+1) \tau} y(t) d t=\infty
$$

which contradicts the fact that $y \in L^{1}\left[t_{0}, \infty\right)$. Thus (2) holds. 
Now we prove that $\lim _{t \rightarrow \infty} y(t)=0$. Since $\sum_{i=1}^{\infty}|z(t+i \tau)|$ converges uniformly on $\left[t_{0}, t_{0}+2 \tau\right]$ and $0<-1 / p<1$; for arbitrary $\varepsilon>0$ there is a positive integer $N$ such that

$$
\sum_{i=k+1}^{k+s}\left(-\frac{1}{p}\right)^{i-k}|z(t+i \tau)|<\frac{\varepsilon}{2}
$$

for all $k \geq N$, all positive integers $s$ and all $t \in\left[t_{0}, t_{0}+2 \tau\right]$. Since $z(t)$ is strictly increasing and $z(t)<0,|z(t+i \tau)|=-p y(t+(i-1) \tau)-y(t+i \tau)$ and the inequality above reduces to

$$
0<y(t+k \tau)-\left(-\frac{1}{p}\right)^{s} y(t+(k+s) \tau)<\frac{\varepsilon}{2}
$$

for all natural numbers $s$. Now let $s \rightarrow \infty$. We get $0<y(t+k \tau)<\varepsilon$ for all $k \geq N$ and all $t \in\left[t_{0}, t_{0}+2 \tau\right]$. Thus, $\lim _{k \rightarrow \infty} y(t+k \tau)=0$ uniformly on $\left[t_{0}, t_{0}+2 \tau\right]$. But that implies that $\lim _{t \rightarrow \infty} y(t)=0$, and the conjecture is proved for this case.

Finally, for the case that $q>0$ and $\tau<0$, set $\tau^{*}=-\tau>0, p^{*}=1 / p$, $q^{*}=q / p<0$ and $\sigma^{*}=\sigma-\tau$. Then the result follows directly from Lemma 3 and the case just proved. Thus, the proof of Conjecture 1 is completed.

\section{Discussion of Conjecture 2.}

Theorem 2. Suppose $p=1, q<0$ and $\tau \neq 0$. Let $y(t)$ be $a$ nonoscillatory solution of (1). Then

$$
\lim _{t \rightarrow \infty} y(t)=-\infty \quad \text { or } \quad \lim _{t \rightarrow \infty} y(t)=\infty
$$

Proof. Without loss of generality, we may suppose that the solution $y(t)$ of $(1)$ is positive for $t \geq t_{0}$. Pick $T>t_{0}$ such that $y(t)>0$ for $t \geq T-|\sigma|$. Since $y(t)$ is continuous, it is uniformly continuous on $[T-|\sigma|, T+2 \tau]$. Hence, $y(t)$ has a minimum $m>0$ on $[T-|\sigma|, T+2 \tau]$. Let $z(t):=y(t)+y(t-\tau)$. Then $z^{\prime}(t)=-q y(t-\sigma)>0$ for $t \geq T$. From Lemma 1, $z(t)$ increases monotonically to $\infty$ as $t \rightarrow \infty$. 
Suppose that $\tau>0$. For $t \in[T, T+2 \tau]$ and every positive integer $n$ we have

$$
y(t+2 n \tau)-y(t+2(n-1) \tau)=z(t+2 n \tau)-z(t+(2 n-1) \tau)>0 .
$$

Consequently, $y(t+2 n \tau)>y(t) \geq m$. Also, we have

$$
\begin{aligned}
z(t+2 n \tau)-z(t+(2 n-1) \tau) & =\tau z^{\prime}\left(t_{n}+(2 n-1) \tau\right) \\
& =-\tau q y\left(t_{n}+(2 n-1) \tau-\sigma\right) \\
& \geq-\tau q m
\end{aligned}
$$

where $t_{n} \in[T, T+2 \tau]$. Then

$$
\begin{aligned}
y(t+2 n \tau)-y(t) & =\sum_{j=1}^{n}[y(t+2 j \tau)-y(t+2(j-1) \tau)] \\
& =\sum_{j=1}^{n}[z(t+2 j \tau)-z(t+(2 j-1) \tau)] \\
& \geq(-q) \tau m n
\end{aligned}
$$

for each positive integer $n$. Now for any $M>0$, let $H:=T+$ $2([-M /(\tau q m)]+1) \tau$. If $t \geq H$, then $t=t^{\prime}+2 n \tau$, where $t^{\prime} \in[T, T+2 \tau]$ and $n$ is a positive integer, so that $y(t)=y\left(t^{\prime}+2 n \tau\right)>(-q) \tau q m n \geq M$. Therefore, $\lim _{t \rightarrow \infty} y(t)=\infty$.

For the case $p=1$ and $\tau<0$, the result follows by setting $\tau^{*}=-\tau$ and applying Lemma 3 and the result just proved. Hence, Theorem 1 has been proved.

The two cases remaining in Conjecture 2, namely, $p>1, q<0, \tau>0$ and $0<p<1, q<0, \tau<0$, are equivalent by Lemma 3 , so we will discuss only the former.

Lemma 4. Let $p>1, q<0$ and $\tau>0$. if $y(t)$ is an eventually positive solution of NFDE (1), then $\lim \sup _{t \rightarrow \infty} y(t)=\infty$.

Proof. Let $z(t):=y(t)+p y(t-\tau)$. Then $z(t)$ is monotonically increasing with limit $\infty$ as $t$ tends to $\infty$. Then clearly $y(t)$ must be unbounded as $t$ tends to $\infty$, so the lemma holds. 
Next observe that $z(t)=y(t)+p y(t-\tau)$ and $z^{\prime}(t)=-q y(t-\sigma)$ together imply that $z^{\prime}(t) \leq-q z(t-\sigma)$ and $z^{\prime}(t) \leq(-q / p) z(t+\tau-\sigma)$. From the first of these two inequalities we get

$$
z^{\prime}(t) \leq-q z(t) \quad \text { if } \sigma \geq 0
$$

while from the second we get

$$
z^{\prime}(t) \leq-\frac{q}{p} z(t) \quad \text { if } \sigma \geq \tau
$$

From (6) we get $e^{-q \tau} z(t-\tau) \geq z(t)$. Hence

$$
\begin{aligned}
p^{2} y(t-2 \tau)-y(t) & =p z(t-\tau)-z(t) \\
& =\left(p-e^{-q \tau}\right) z(t-\tau)+e^{-q \tau} z(t-\tau)-z(t) \\
& \geq\left(p-e^{-q \tau}\right) z(t-\tau) \rightarrow \infty
\end{aligned}
$$

as $t \rightarrow \infty$ in case

$$
p>e^{-q \tau} \quad \text { and } \quad \sigma \geq 0
$$

If we use inequality (7) we get

$$
e^{-q \tau / p} z(t-\tau) \geq z(t)
$$

so that $p^{2} y(t-2 \tau)-y(t) \rightarrow \infty$ as $t \rightarrow \infty$, provided

$$
p \ln p>-q \tau \quad \text { and } \quad \sigma \geq \tau
$$

Also observe that $z(t)$ solves NFDE (1). Then integration from $t$ to $t+\tau$ gives

$$
z(t+\tau)+p z(t)-z(t)-p z(t-\tau) \leq-q \tau z(t+\tau) \quad \text { if } \sigma \geq 0
$$

Thus

$$
\begin{aligned}
p^{2} y(t-2 \tau)-y(t) & =p[z(t-\tau)-z(t)]+(p-1) z(t) \\
& \geq z(t+\tau)-z(t)+q \tau z(t+\tau)+(p-1) z(t) \\
& =(1+q \tau) z(t+\tau)+(p-2) z(t) \rightarrow \infty
\end{aligned}
$$




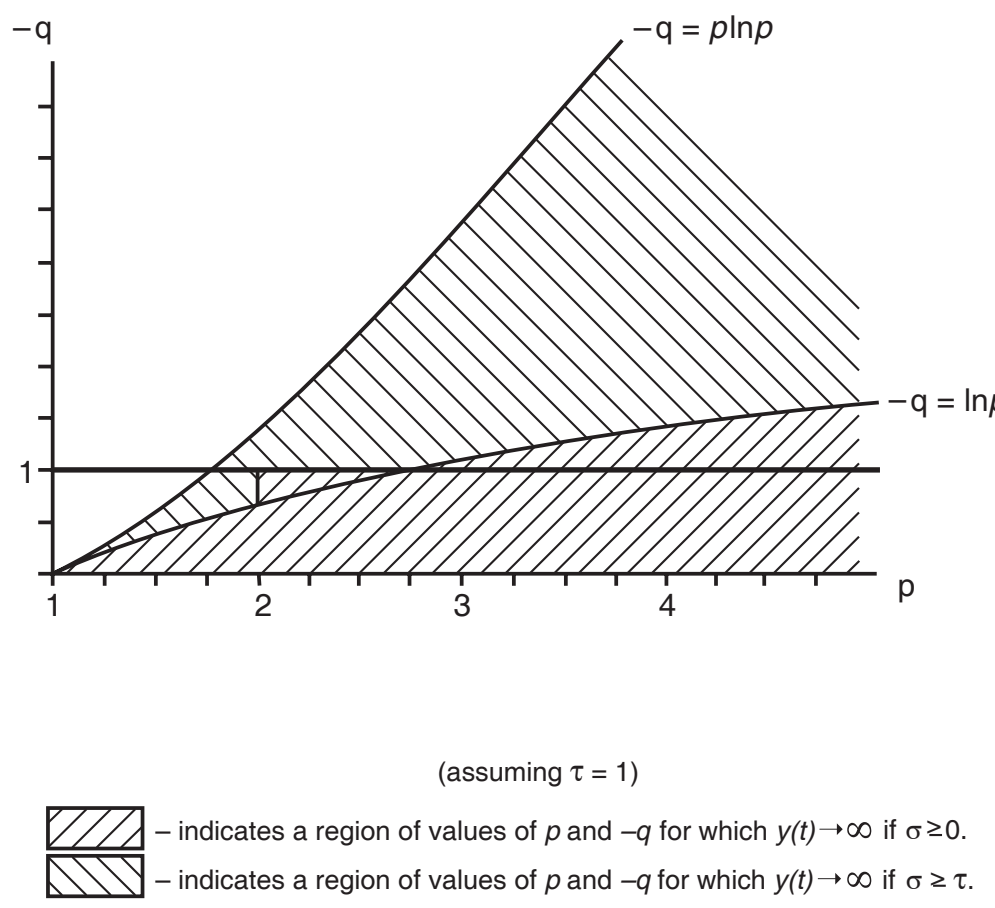

FIGURE 1. Regions for Theorem 3 (assuming $\tau=1$ ).

as $t \rightarrow \infty$, provided

(10) $\sigma>0,1+q \tau \geq 0, p-2 \geq 0$ and one of the last two is strict.

We collect these observations as a theorem.

Theorem 3. Let $p>1, q<0, \tau>0$. Then any one of the conditions (8), (9), or (10) is a sufficient condition for every eventually positive solution of NFDE (1) to tend to $\infty$ as $t \rightarrow \infty$.

Figure 1 indicates there are still many values of $p$ and $q$ for which Conjecture 2 remains unresolved. In particular, there are no results 


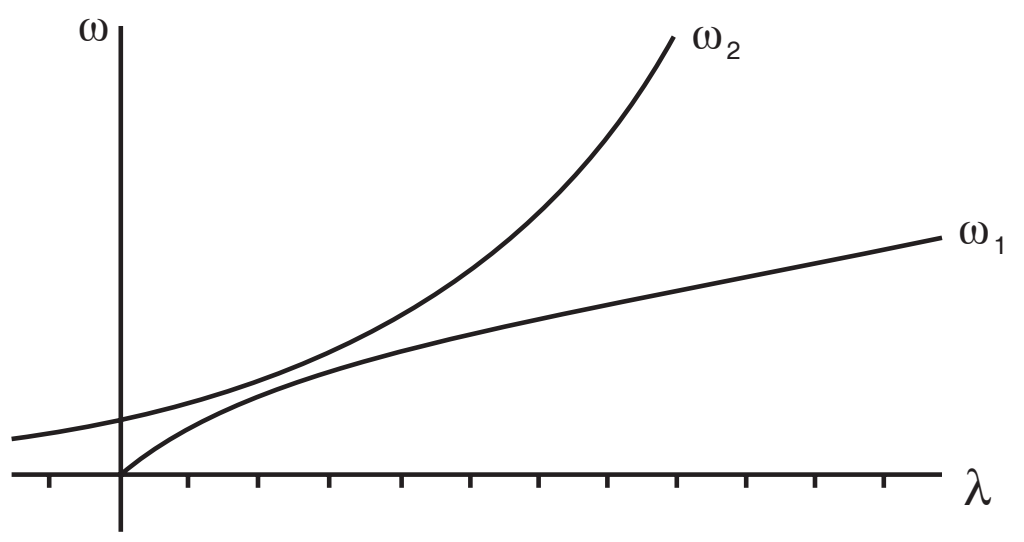

FIGURE 2. Characteristic Equation-no real roots.

given above for the case that $\sigma<0$. It is known that NFDE (1) has only oscillatory solutions if and only if its characteristic equation

$$
\lambda+p \lambda e^{-\tau \lambda}+q e^{-\sigma \lambda}=0
$$

has no real solutions. See [6] as well as $[\mathbf{1}, \mathbf{9}$ and 10] for the proof of this result in other cases. Clearly, (11) has a real solution if and only if the graphs of $\omega=\omega_{1}(\lambda):=\lambda\left(1+p e^{-\tau \lambda}\right)$ and $\omega=\omega_{2}(\lambda):=-q e^{-\sigma \lambda}$ have a point of intersection. If $p>0, q<0, \tau>0$ and $\sigma>0$, such a point always exists. For $\sigma<0$, the situation is different as is illustrated in Figure 2. For $-q$ large, the graphs do not intersect, but for $-q$ small and positive, there are two points of intersection. It follows that there is a largest value $-q_{0}$ of $-q$ for which $\omega_{1}(\lambda)=\omega_{2}(\lambda)$ has a (unique) solution. To find $-q_{0}$, note that $-q_{0}=\lambda\left(1+p e^{-\tau \lambda}\right) e^{\sigma \lambda}$ for some $\lambda=\lambda_{0}$ and maximize this expression in $\lambda$. It is straightforward to show that there is a unique such $\lambda_{0}$ which must satisfy $1 /(t-\sigma)<\lambda_{0}<-1 / \sigma$ and solve the equation

$$
p e^{-t \lambda_{0}}=\frac{\sigma \lambda_{0}+1}{(t-\sigma) \lambda_{0}-1} .
$$

Now for $-q>-q_{0},(11)$ has no real roots and consequently (1) has only oscillatory solutions. We state this result as a theorem. 
Theorem 4. Suppose $p>0, \tau>0, \sigma<0$ and $q<0$. Let $\lambda_{0}$ be the unique solution of (12). Then NFDE (1) has only oscillatory solutions if and only if

$$
-q>\lambda_{0}\left(1+p e^{-\tau \lambda_{0}}\right) e^{\sigma \lambda_{0}}
$$

Corollary. Suppose $p>0, \tau>0, q<0$ and $\sigma<0$. Then (1) has only oscillatory solutions in case

$$
q \sigma>e^{2 \sigma /(\tau-2 \sigma)}+p e^{2(\sigma-\tau) /(\tau-2 \sigma)} .
$$

Proof. Since $\lambda_{0} \in(1 /(\tau-\sigma), 1 /(-\sigma))$, it lies in one of the two intervals $(1 /(\tau-\sigma), 2 /(\tau-2 \sigma)]$ and $[2 /(\tau-2 \sigma), 1 /(-\sigma))$. On the first of these intervals we have

$$
\lambda\left(1+p e^{-\tau \lambda}\right) e^{\sigma \lambda} \leq \frac{1}{\tau-2 \sigma}\left(e^{2 \sigma /(\tau-2 \sigma)}+\frac{p}{e}\right)
$$

and on the second a bound is $(1 /(-\sigma))\left(e^{2 \sigma /(\tau-2 \sigma)}+p e^{2(\sigma-\tau) /(\tau-2 \sigma)}\right)$. The second bound is the larger if and only if

$$
e^{\tau /(\tau-2 \sigma)}+p e^{-\tau /(\tau-2 \sigma)}>\frac{-2 \sigma}{\tau-2 \sigma}\left(e^{\tau /(\tau-\sigma)}+p\right)
$$

which is equivalent to

$$
p\left(e^{-\tau /(\tau-2 \sigma)}+\frac{2 \sigma}{\tau-2 \sigma}\right)>\frac{-2 \sigma}{\tau-2 \sigma} e^{\tau /(\tau-\sigma)}-e^{\tau /(\tau-2 \sigma)} .
$$

Let $\tau=-k \sigma$. The inequality then becomes

$$
p\left(e^{k /(k+2)}-\frac{2}{k+2}\right)>\frac{2}{k+2} e^{k /(k+1)}-e^{k /(k+2)} .
$$

The left side of this inequality clearly is positive. The right side can be rewritten as

$$
\frac{2 e^{k /(k+2)}}{k+2}\left(e^{k /(k+1)(k+2)}-\frac{k+2}{2}\right) \text {. }
$$


Now use the fact that $e^{r}<1+r+3 r^{2} /[2(3-r)]$ for $0<r<1$ to show that the right side is negative. Hence the second bound is larger. Thus, if (14) holds, so does (13). The lemma is proved.

If the sufficient condition for oscillation developed by Schultz in [9] is specialized to NFDE (1), the result is $q \sigma e>1+p$. On the other hand (14) can be rewritten as $q \sigma e>e^{k /(k+2)}+p e^{-k /(k+2)}$. Now $1+p \geq e^{k /(k+2)}+p e^{-k /(k+2)}$ if and only if $p \geq e^{k /(k+2)}$. Since $k>0$, if $p \geq e$, (14) gives a smaller bound and hence gives the oscillation of all solutions of (1) for more values of $q$. As an example, consider the equation

$$
\frac{d}{d t}[y(t)+2 y(t-1)]+q y(t+1)=0 .
$$

Here $p=2$ and $\tau=-\sigma=1$. Schultz's condition gives oscillation if $-q>3 / e$ while $(14)$ requires $-q>e^{-2 / 3}+2 e^{-4 / 3} \doteq 1.0406<3 / e \doteq$ 1.1036. Condition (13) for this example gives $-q>-q_{0} \doteq .6938$, a much better bound as one expects.

5. Conclusions and examples. We have now fully resolved six of the eight cases conjectured in [5]. In the remaining two equivalent cases we have given additional restrictions on the coefficients under which Conjecture 2 is correct. The counterexamples in [8], which show that in general Conjecture 2 is false, lie in the $-q, p$ plane along the curve $-q=2 p \ln p$ for $\sigma=1=\tau$ and along $-q=2 \ln p$ for $\tau=1>0=\sigma$. What remains is an analysis of the asymptotic behavior of the nonoscillatory solutions of NDDE (1) in the cases not covered by Theorem 3. For example, if $\sigma \geq \tau$ and $p \ln p<-q<2 p \ln p$ or if $\tau>\sigma$ and $p \ln p<-q<2 \ln p$, the results given above do not apply.

It is worth noting that in Conjecture 1 the solutions of (1), even the $C^{\infty}$ solutions, satisfying

$$
\lim _{t \rightarrow \infty} y(t)=-\infty, \quad \lim _{t \rightarrow \infty} y(t)=0 \quad \text { or } \quad \lim _{t \rightarrow \infty} y(t)=\infty
$$

can occur in the same equation. For example, the NFDEs

$$
\frac{d}{d t}\left[y(t)-\frac{20}{33} y(t+\ln 2)\right]+\frac{14}{33} y(t-\ln 2)=0, \quad 0>p>-1, q>0, \tau<0
$$


and

$$
\frac{d}{d t}\left[y(t)-\frac{5}{17} y(t-\ln 4)\right]-\frac{63}{34} y(t-\ln 2)=0, \quad 0>p>-1, q<0, \tau>0
$$

both have nonoscillatory solutions $\pm e^{-2 t}$ and $\pm e^{+t}$; the NFDEs

$$
\frac{d}{d t}[y(t)-y(t+\ln 4)]+\frac{3}{2} y(t+\ln 2)=0, \quad p=-1, q>0, \tau<0
$$

and

$$
\frac{d}{d t}[y(t)-y(t-\ln 4)]-\frac{3}{2} y(t-\ln 2)=0, \quad p=-1, q<0, \tau<0
$$

both have nonoscillatory solutions $\pm e^{-t}$ and $\pm e^{t}$; the NFDEs

$$
\frac{d}{d t}\left[y(t)-\frac{17}{5} y(t+\ln 4)\right]+\frac{63}{10} y(t+\ln 2)=0, \quad p<-1, q>0, \tau<0
$$

and

$$
\frac{d}{d t}\left[y(t)-\frac{33}{20} y(t-\ln 2)\right]-\frac{7}{10} y(t-\ln 4)=0, \quad p<-1, q<0, \tau>0
$$

both have nonoscillatory solutions $\pm e^{-2 t}$ and $\pm e^{t}$. Actually, there is an infinite collection of such equations. For instance, given an arbitrary $p \in\left(-e^{1 / \alpha},-e^{-1}\right)$ and an arbitrary $\alpha>0$, the NFDEs

$$
\frac{d}{d t}[y(t)+p y(t-1 / \alpha)]+q y(t-\sigma)=0
$$

and

$$
\frac{d}{d t}\left[y(t)+\frac{1}{p} y(t+1 / \alpha)\right]+\frac{q}{p} y(t+1 / \alpha-\sigma)=0
$$

where

$$
\sigma=\frac{1}{1+\alpha} \ln \left[\frac{\alpha(1+p e)}{-1-p e^{-1 / \alpha}}\right]
$$

and

$$
q=\alpha(1+p e)\left[\frac{\alpha(1+p e)}{-1-p e^{-1 / \alpha}}\right]^{-\alpha /(\alpha+1)}<0
$$


always have nonoscillatory solutions $\pm e^{\alpha t}$ and $\pm e^{t}$.

\section{REFERENCES}

1. O. Arino and I. Györi, Necessary and sufficient conditions for oscillation of a neutral differential system with several delays, J. Differential Equations 81 (1989), 98-105.

2. R.K. Brayton and R.A. Willoughby, On the numerical integration of a symmetric system of difference-differential equations of neutral type, J. Math. Anal. Appl. 18 (1967), 182-189.

3. R.D. Driver, A mixed neutral system, Nonlinear Anal. 8 (1984), 155-158.

4. - Existence and continuous dependence of solutions of a neutral functional differential equation, Arch. Rat. Mech. Anal. 19 (1965), 149-166.

5. M.K. Grammatikopoulos, E.A. Grove and G. Ladas, Oscillation and asymptotic behavior of neutral differential equations with deviating arguments, Appl. Anal. 22 (1986), 1-19.

6. E.A. Grove, G. Ladas and A. Meimaridou, A necessary and sufficient condition for the oscillation of neutral equations, J. Math. Anal. Appl. 126 (1987), 341-354.

7. J. Hale, Theory of functional differential equations, Springer-Verlag, New York 1977.

8. T. Krisztin, R.M. Mathsen and Xu Yuantong, Counter examples to a conjecture for neutral equations, Canad. Math. Bull. 36(1) (1993), 74-77.

9. S.W. Schultz, Necessary and sufficient conditions for oscillation of neutral equations with mixed argument, in Differential equations and applications: Proceedings of the int. conf. on theory and appl. of diff. eqns., Columbus, Ohio, 1988, Ohio University Press, Athens, 1989.

10. Y.G. Sficas and I.P. Stavroulakis, Necessary and sufficient conditions for oscillation of neutral equations, J. Math. Anal. Appl. 123 (1987), 494-507.

11. M. Slemrod and E.F. Infante, Asymptotic stability criteria for linear systems of difference-differential equations of neutral type and their discrete analogues, J. Math. Anal. Appl. 38 (1972), 399-415.

12. Xu Yuantong, Asymptotic behavior of nonoscillatory solutions of higher-order neutral equations, Ann. Diff. Eqns. 5 (1989), 199-209.

Department of Mathematics, North Dakota State University, Fargo, ND $58105-5075$

Department of Mathematics, Zhongshan University, Guangzhou, 510275 P.R. China

Department of Mathematics, Zhongshan University, Guangzhou, 510275 P.R. ChINA 